\title{
V752 Cen - A triple-lined spectroscopic contact binary with sudden and continuous period changes
}

\author{
X. Zhou ${ }^{1,2,3,4} \star$, B. Soonthornthum², S.-B. Qian ${ }^{1,3,4,5}$, E. Fernández, Lajús ${ }^{6,7}$ \\ 1 Yunnan Observatories, Chinese Academy of Sciences (CAS), P.O. Box 110, 650216 Kunming, China \\ 2 National Astronomical Research Institute of Thailand, 260 Moo 4, T. Donkaew, A. Maerim, Chiangmai, 50180, Thailand \\ ${ }^{3}$ Key Laboratory of the Structure and Evolution of Celestial Objects, Chinese Academy of Sciences, P. O. Box 110, 650216 Kunming, China \\ 4 Center for Astronomical Mega-Science, Chinese Academy of Sciences, 20A Datun Road, Chaoyang District, Beijing, 100012, China \\ 5 University of the Chinese Academy of Sciences, Yuquan Road 19\#, Sijingshang Block, 100049 Beijing, P. R. China \\ ${ }^{6}$ Facultad de Ciencias Astronómicas y Geofísicas, Universidad Nacional de La Plata, Paseo del Bosque s/n, 1900, La Plata, Pcia. Bs. As., \\ Argentina \\ 7 Instituto de Astrofísica de La Plata (CCT La plata - CONICET/UNLP), Argentina
}

16 September 2019

\begin{abstract}
V752 Cen is a triple-lined spectroscopic contact binary. Its multi-color light curves were obtained in the years 1971 and 2018, independently. Photometric analyses reveal that the two sets of light curves produce almost consistent results. It contains a Wsubtype totally eclipsing binary, and its mass ratio and fill-out factor are $q=3.35(1)$ and $f=29(2) \%$. The absolute elements of its two component stars were determined to be $M_{1}=0.39(2) M_{\odot}, M_{2}=1.31(7) M_{\odot}, R_{1}=0.77(1) R_{\odot}, R_{2}=1.30(2) R_{\odot}, L_{1}=$ $0.75(3) L_{\odot}$ and $L_{2}=2.00(7) L_{\odot}$. The period of V752 Cen is 0.37023198 day. The 0.37-d period remained constant from its first measurement in 1971 until the year 2000. However, it changed suddenly around the year 2000 and has been increasing continuously at a rate of $d P / d t=+5.05 \times 10^{-7} d a y \cdot y e a r^{-1}$ since then, which can be explained by mass transfer from the less massive component star to the more massive one with a rate of $\frac{d M_{2}}{d t}=2.52 \times 10^{-7} M_{\odot} /$ year. The period variation of V752 Cen over the 48 years in which the period has been monitored is really unusual, and is potentially related to effects from the possible presence of a nearby third star or of a pair of stars in a second binary.
\end{abstract}

Key words: techniques: photometric — binaries: eclipsing — stars: individual (V752 Cen)

\section{INTRODUCTION}

Eclipsing binaries have proved to be powerful probes for studying a wide range of astrophysical problems. They are the primary sources in providing fundamental stellar parameters such as mass, radius and luminosity, which are very important in testing theoretical models of stellar evolution (Soszyński et al. 2016). Over the past decades, the number of known eclipsing binaries has increased rapidly. Photometric light curves of eclipsing binaries are archived by many survey projects, such as the Optical Gravitational Lensing Experiment (OGLE) (Graczyk et al. 2011; Rucinski 1997), the All Sky Automated Survey (ASAS) (Javasinghe et al. 2019) and the Kepler Space Telescope (Prša et al. 2011; Slawson et al. 2011). What is even more exciting is that many spectroscopic survey projects are carried out at the same time, such as the Sloan Digital Sky Survey (SDSS) (York et al. 2000), the Large Sky Area Multi-Object Fibre Spectroscopic Telescope (LAMOST) (Qian et al. 2017, 2018) and the Gaia mission (Gaia Collaboration et al. 2016, 2018). This means that many eclipsing binaries have been observed by both photometric and spectroscopic methods.

* E-mail: zhouxiaophy@ynao.ac.cn 
Table 1. Newly determined mid-eclipse times.

\begin{tabular}{ccccc}
\hline \hline JD (Hel.) & Error (days) & $\mathrm{p} / \mathrm{s}$ & Filter & Method \\
\hline \hline 2458224.6073 & \pm 0.0001 & $\mathrm{p}$ & $V$ & $\mathrm{CCD}$ \\
2458224.7936 & \pm 0.0001 & $\mathrm{~s}$ & $V$ & $\mathrm{CCD}$ \\
2458225.5337 & \pm 0.0001 & $\mathrm{~s}$ & $R_{C}$ & $\mathrm{CCD}$ \\
2458225.7181 & \pm 0.0001 & $\mathrm{p}$ & $R_{C}$ & $\mathrm{CCD}$ \\
2458226.6443 & \pm 0.0001 & $\mathrm{~s}$ & $I_{C}$ & $\mathrm{CCD}$ \\
2458227.5692 & \pm 0.0001 & $\mathrm{p}$ & $B$ & $\mathrm{CCD}$ \\
2458227.7551 & \pm 0.0001 & $\mathrm{~s}$ & $B$ & $\mathrm{CCD}$ \\
2458228.4956 & \pm 0.0001 & $\mathrm{~s}$ & $V$ & $\mathrm{CCD}$ \\
2458228.6803 & \pm 0.0001 & $\mathrm{p}$ & $V$ & $\mathrm{CCD}$ \\
2458642.6058 & \pm 0.0001 & $\mathrm{p}$ & $I_{C}$ & $\mathrm{CCD}$ \\
\hline \hline
\end{tabular}

The observations on contact binaries have a long history. Almost all identified contact binaries belong to eclipsing binaries. However, the formation of contact binaries is still an open issue. Some researchers claim that contact binaries evolve from low-mass detached close binaries via the loss of angular momentum by means of a magnetic wind (Tutukov et al. 2004; Ylldız \& Doğan 2013). Another hypothesis is that tertiary components have played a very important role in the formation of contact binaries by removing angular momentum from the central binaries (Qian et al. 2013, 2014). Tertiary components are really common in contact binaries and almost every kind of celestial body can be a tertiary component orbiting the central binary, from planets to black holes (Xiao et al. 2016). Tokovinin et al. (2006) conclude that $96 \%$ of binaries with periods shorter than 3 days have tertiary components, based on a survey of 165 solar-type spectroscopic binaries. What is more, some contact binaries even have close-in companions with distance less than 3 AU (Zhou et al. 2017), and contact binaries with more than one companions were also reported (Zhu et al. 2013; Li et al. 2015). All of these hierarchical contact binaries are very important samples for investigating the dynamic interactions among multi-stellar systems.

In 1970, Bond (1970) reported three newly discovered W UMa type contact binaries, one of which was V752 Cen (HD 101799, $\left.V=9.1^{m}\right)$. Later, Sisteró \& Castore de Sisteró $(1971$, 1973) observed V752 Cen photometrically and obtained $U B V$ light curves. They claimed that V752 Cen was a completely eclipsing binary system and its components nearly filled their respective Roche lobes (a semi-detached system). The radial velocity curves of V752 Cen were also published by Sisteró \& Castore de Sisteró (1974), which revealed that V752 Cen was a double-lined eclipsing binary with the spectral type of its two component stars to be F8 and F5. However, Leung (1976) claimed that V752 Cen was a contact binary with a fill-out factor of $f=6.3 \%$ after he reanalyzed the light curves obtained by Sisteró \& Castore de Sisteró (1971, 1973). Barone et al. (1993) also supported the contact configuration with a little higher fill-out factor (9\%). The fill-out factor is defined as $f=\frac{\Omega_{\text {in }}-\Omega_{1,2}}{\Omega_{\text {in }}-\Omega_{\text {out }}}$, where $\Omega_{\text {in }}$ and $\Omega_{\text {out }}$ are the potentials at the inner and outer Lagrangian points of a binary system, and $\Omega_{1,2}$ are the surface potentials of the component stars (Ruciński 1973). The high resolution spectroscopy of V752 Cen found that it may be a triple-lined spectroscopic quadruple system (Schumacher 2009). Mallama \& Pavlov (2015) pointed out that the orbital period of V752 Cen was not always constant, and there were some small magnitude changes around the time that the period was inferred to change. V752 Cen was also listed in the Gaia Data Release 2 (DR2) with effective temperature to be $T_{1}=6138 \mathrm{~K}$ and parallax to be 7.96mas (Gaia Collaboration et al. 2016, 2018).

In the present work, we are going to reanalyze the light curves of V752 Cen published in April 1971 and the newly obtained ones in April 2018 to derive the physical parameters of the primary star and the secondary one. And also, its period variations over the past several decades will be revealed. Research on this hierarchical stellar system will provide valuable information about the evolution of hierarchical contact binary systems.

\section{PHOTOMETRIC OBSERVATIONS}

Photometric observations of V752 Cen were carried out at the Complejo Astronomico El Leoncito (CASLEO), San Juan, Argentina, with the 0.60 m Helen Sawyer Hogg (HSH) Telescope on 2018 April 15 - 19. Johnson-Cousins $B V R_{c} I_{c}$ filters were used during the observations. However, only one filter was used each night. The $V$ filter was used on 2018 April 15 and 19 , and the $R_{c}, I_{c}$ and $B$ filters were used on 2018 April 16, 17 and 18, respectively. The observational light curves are displayed in Fig. 1 In order to get more minima times and cover a longer time span in the $\mathrm{O}$ - C diagram, V752 Cen was observed again with the $I_{c}$ filter on 2019 June 7 . A total of ten mid-eclipse times of V752 Cen were determined, which are listed in Table 1 .

\section{INVESTIGATION OF PERIOD VARIATIONS}

Period variations commonly appear in close binary systems, especially for contact binaries, due to the possible mass transfer between their component stars or angular momentum loss from the binary systems. V752 Cen is a short period eclipsing 


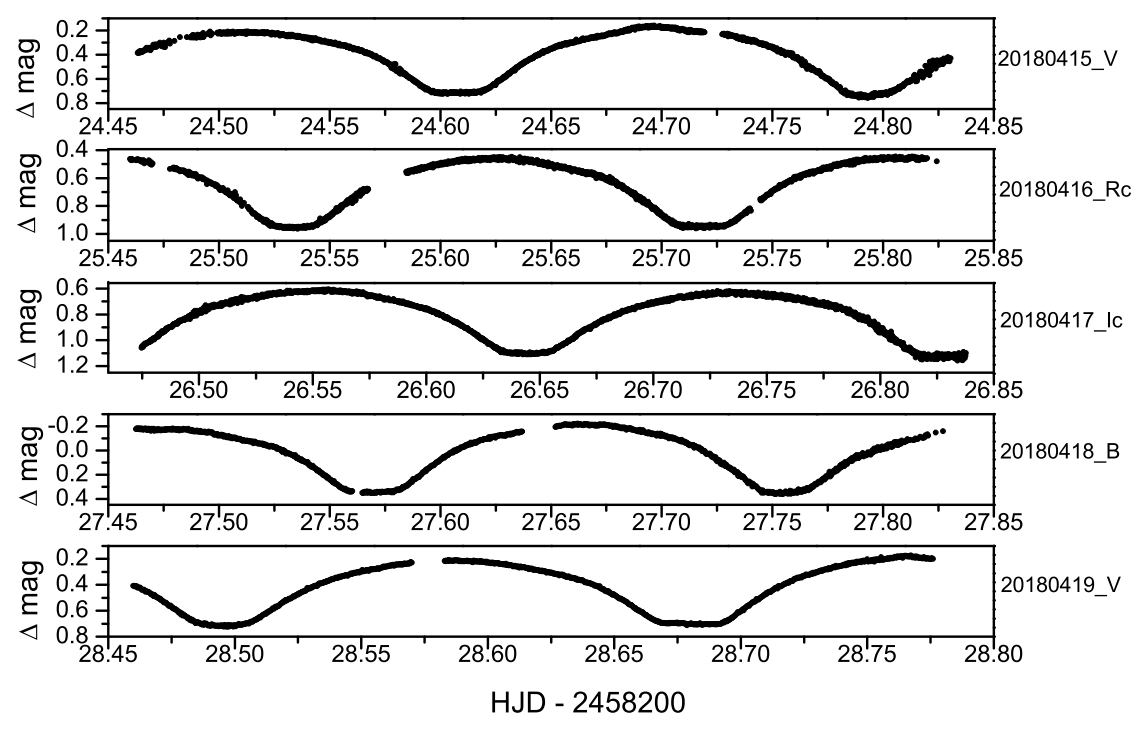

Figure 1. Observational light curves of V752 Cen. The yellow, red, green and blue colors refer to $V, R_{c}, I_{c}$ and $B$ filters respectively.

binary system, which is listed in the International Variable Star Index (VSX) 11 as having a period P $=0.37023198$ days. In the present work, all published mid-eclipse times of V752 Cen were collected to investigate its period variations. And also, V752 Cen was observed by the SuperWASP project (Butters et al. 2010) and the Transiting Exoplanet Survey Satellite (TESS)(Ricker et al. 2015). The observational light curves were downloaded and 84 mid-eclipse times were determined from SuperWASP data. V752 Cen was observed by TESS from 2019 March 26 to 2019 April 22 continuously and 120 times of minima were determined (see Table 2). The minima in HJD were converted to BJD since the minima determined in TESS data used BJD time system. The linear equation used to calculate the $O-C$ values is:

$$
\operatorname{Min} . I(B J D)=2456108.3448+0^{d} .37023198 \times E .
$$

Column 1 - Heliocentric Julian Date of the observed mid-eclipse times (HJD - 2400000);

Column 2 - Barycentric Julian Date of the observed mid-eclipse times (BJD - 2400000);

Column 3 - primary (p) or secondary (s) mid-eclipse times;

Column 4 - PE, Vis and CCD refer to photoelectric, visual and Charge Coupled Device observations;

Column 5 - error of mid-eclipse times;

Column 6 - cycle numbers from the initial epoch;

Column 7 - the $O-C$ values calculated from Equation 1

Column 8 - the references;

Table 2: Mid-eclipse times and $O-C$ values for V752 Cen (the whole table is available in the online journal).

\begin{tabular}{llllllll}
\hline \hline $\begin{array}{l}\text { HJD } \\
(2400000+)\end{array}$ & $\begin{array}{l}\text { BJD } \\
(2400000+)\end{array}$ & $\mathrm{p} / \mathrm{s}$ & Method & $\begin{array}{l}\text { Error } \\
\text { (days) }\end{array}$ & Epoch & $\begin{array}{l}O-C \\
\text { (days })\end{array}$ & Ref. \\
\hline 40648.613 & 40648.6135 & $\mathrm{~s}$ & $\mathrm{PE}$ & & -41757.5 & 0.2306 & 1 \\
40648.98393 & 40648.9844 & $\mathrm{~s}$ & $\mathrm{PE}$ & & -41756.5 & 0.2313 & 2 \\
41056.60633 & 41056.6068 & $\mathrm{~s}$ & $\mathrm{PE}$ & & -40655.5 & 0.2283 & 2 \\
41056.60640 & 41056.6069 & $\mathrm{~s}$ & $\mathrm{PE}$ & & -40655.5 & 0.2284 & 2 \\
41056.60652 & 41056.6070 & $\mathrm{~s}$ & $\mathrm{PE}$ & & -40655.5 & 0.2285 & 2 \\
41057.53144 & 41057.5319 & $\mathrm{p}$ & $\mathrm{PE}$ & & -40653 & 0.2278 & 2 \\
41057.53191 & 41057.5324 & $\mathrm{p}$ & $\mathrm{PE}$ & & -40653 & 0.2283 & 2 \\
41057.53220 & 41057.5327 & $\mathrm{p}$ & $\mathrm{PE}$ & & -40653 & 0.2286 & 2 \\
41057.71732 & 41057.7178 & $\mathrm{~s}$ & $\mathrm{PE}$ & & -40652.5 & 0.2286 & 2 \\
\hline \hline
\end{tabular}

1 https://www.aavso.org/vsx/ 
X. Zhou et. al

Table 2 continued

\begin{tabular}{|c|c|c|c|c|c|c|c|}
\hline $\begin{array}{l}\text { HJD } \\
(2400000+)\end{array}$ & $\begin{array}{l}\text { BJD } \\
(2400000+)\end{array}$ & $\overline{\mathrm{p} / \mathrm{s}}$ & $\overline{\text { Method }}$ & $\begin{array}{l}\begin{array}{l}\text { Error } \\
(\text { days })\end{array} \\
\text { (d) }\end{array}$ & $\overline{\text { Epoch }}$ & $\begin{array}{l}O-C \\
\text { (days) }\end{array}$ & $\overline{\text { Ref. }}$ \\
\hline 41057.71742 & 41057.7179 & $\mathrm{~s}$ & $\mathrm{PE}$ & & -40652.5 & 0.2287 & 2 \\
\hline 41057.71749 & 41057.7180 & $\mathrm{~s}$ & $\mathrm{PE}$ & & -40652.5 & 0.2287 & 2 \\
\hline 41058.64242 & 41058.6429 & $\mathrm{p}$ & $\mathrm{PE}$ & & -40650 & 0.2281 & 2 \\
\hline 41058.64279 & 41058.6433 & $\mathrm{p}$ & $\mathrm{PE}$ & & -40650 & 0.2285 & 2 \\
\hline 41058.64353 & 41058.6440 & $\mathrm{p}$ & $\mathrm{PE}$ & & -40650 & 0.2292 & 2 \\
\hline 41059.56740 & 41059.5679 & $\mathrm{~s}$ & $\mathrm{PE}$ & & -40647.5 & 0.2275 & 2 \\
\hline 41059.56798 & 41059.5684 & $\mathrm{~s}$ & $\mathrm{PE}$ & & -40647.5 & 0.2281 & 2 \\
\hline 41059.56809 & 41059.5686 & $\mathrm{~s}$ & $\mathrm{PE}$ & & -40647.5 & 0.2282 & 2 \\
\hline 41059.75328 & 41059.7537 & $\mathrm{p}$ & $\mathrm{PE}$ & & -40647 & 0.2283 & 2 \\
\hline 41059.75350 & 41059.7540 & $\mathrm{p}$ & $\mathrm{PE}$ & & -40647 & 0.2285 & 2 \\
\hline 41059.75370 & 41059.7542 & $\mathrm{p}$ & $\mathrm{PE}$ & & -40647 & 0.2287 & 2 \\
\hline 41060.49273 & 41060.4932 & $\mathrm{p}$ & $\mathrm{PE}$ & & -40645 & 0.2272 & 2 \\
\hline 41060.49285 & 41060.4933 & $\mathrm{p}$ & $\mathrm{PE}$ & & -40645 & 0.2274 & 2 \\
\hline 41060.49292 & 41060.4934 & $\mathrm{p}$ & $\mathrm{PE}$ & & -40645 & 0.2274 & 2 \\
\hline 41060.67820 & 41060.6787 & $\mathrm{~s}$ & $\mathrm{PE}$ & & -40644.5 & 0.2276 & 2 \\
\hline 41060.67834 & 41060.6788 & $\mathrm{~s}$ & $\mathrm{PE}$ & & -40644.5 & 0.2277 & 2 \\
\hline 41060.67877 & 41060.6792 & $\mathrm{~s}$ & $\mathrm{PE}$ & & -40644.5 & 0.2282 & 2 \\
\hline 41061.60298 & 41061.6034 & $\mathrm{p}$ & $\mathrm{PE}$ & & -40642 & 0.2268 & 2 \\
\hline 41061.60383 & 41061.6043 & $\mathrm{p}$ & $\mathrm{PE}$ & & -40642 & 0.2276 & 2 \\
\hline 41061.60395 & 41061.6044 & $\mathrm{p}$ & $\mathrm{PE}$ & & -40642 & 0.2278 & 2 \\
\hline 41063.63958 & 41063.6400 & $\mathrm{~s}$ & $\mathrm{PE}$ & & -40636.5 & 0.2271 & 2 \\
\hline 41063.63972 & 41063.6402 & $\mathrm{~s}$ & $\mathrm{PE}$ & & -40636.5 & 0.2273 & 2 \\
\hline 41063.63973 & 41063.6402 & $\mathrm{~s}$ & $\mathrm{PE}$ & & -40636.5 & 0.2273 & 2 \\
\hline 41064.56504 & 41064.5655 & $\mathrm{p}$ & $\mathrm{PE}$ & & -40634 & 0.2270 & 2 \\
\hline 41064.56545 & 41064.5659 & $\mathrm{p}$ & $\mathrm{PE}$ & & -40634 & 0.2274 & 2 \\
\hline 41064.56676 & 41064.5672 & $\mathrm{p}$ & $\mathrm{PE}$ & & -40634 & 0.2287 & 2 \\
\hline 43244.457 & 43244.4576 & $\mathrm{p}$ & Vis & & -34746 & 0.1932 & 3 \\
\hline 43245.378 & 43245.3786 & $\mathrm{~s}$ & Vis & & -34743.5 & 0.1886 & 3 \\
\hline 43918.649 & 43918.6496 & $\mathrm{p}$ & Vis & & -32925 & 0.1927 & 3 \\
\hline 43937.533 & 43937.5336 & $\mathrm{p}$ & Vis & & -32874 & 0.1949 & 3 \\
\hline 44234.616 & 44234.6165 & $\mathrm{~s}$ & Vis & & -32071.5 & 0.1667 & 3 \\
\hline 44235.547 & 44235.5476 & $\mathrm{p}$ & Vis & & -32069 & 0.1721 & 3 \\
\hline 44243.681 & 44243.6816 & $\mathrm{p}$ & Vis & & -32047 & 0.1610 & 3 \\
\hline 45428.406 & 45428.4066 & $\mathrm{p}$ & Vis & & -28847 & 0.1437 & 3 \\
\hline 45430.456 & 45430.4566 & $\mathrm{~s}$ & Vis & & -28841.5 & 0.1575 & 3 \\
\hline 45431.372 & 45431.3726 & $\mathrm{p}$ & Vis & & -28839 & 0.1479 & 3 \\
\hline 45436.357 & 45436.3576 & $\mathrm{~s}$ & Vis & & -28825.5 & 0.1347 & 3 \\
\hline 45438.400 & 45438.4006 & $\mathrm{p}$ & Vis & & -28820 & 0.1415 & 3 \\
\hline 45753.466 & 45753.4666 & $\mathrm{p}$ & Vis & & -27969 & 0.1401 & 3 \\
\hline 45804.379 & 45804.3796 & $\mathrm{~s}$ & Vis & & -27831.5 & 0.1462 & 3 \\
\hline 45806.391 & 45806.3916 & $\mathrm{p}$ & Vis & & -27826 & 0.1219 & 3 \\
\hline 46162.362 & 46162.3626 & $\mathrm{~s}$ & Vis & & -26864.5 & 0.1149 & 3 \\
\hline 46163.305 & 46163.3056 & $\mathrm{p}$ & Vis & & -26862 & 0.1323 & 3 \\
\hline 46164.417 & 46164.4176 & $\mathrm{p}$ & Vis & & -26859 & 0.1336 & 3 \\
\hline 46166.277 & 46166.2776 & $\mathrm{p}$ & Vis & & -26854 & 0.1424 & 3 \\
\hline 46167.383 & 46167.3836 & $\mathrm{p}$ & Vis & & -26851 & 0.1377 & 3 \\
\hline 46530.385 & 46530.3857 & $\mathrm{~s}$ & Vis & & -25870.5 & 0.1273 & 3 \\
\hline 46535.379 & 46535.3797 & $\mathrm{p}$ & Vis & & -25857 & 0.1232 & 3 \\
\hline 46798.595 & 46798.5957 & $\mathrm{p}$ & Vis & & -25146 & 0.1042 & 3 \\
\hline 46884.321 & 46884.3217 & $\mathrm{~s}$ & Vis & & -24914.5 & 0.1215 & 3 \\
\hline 47262.325 & 47262.3257 & $\mathrm{~s}$ & Vis & & -23893.5 & 0.1187 & 3 \\
\hline 47581.452 & 47581.4527 & $\mathrm{~s}$ & Vis & & -23031.5 & 0.1057 & 3 \\
\hline 47663.272 & 47663.2727 & $\mathrm{~s}$ & Vis & & -22810.5 & 0.1045 & 3 \\
\hline 48008.319 & 48008.3197 & $\mathrm{~s}$ & Vis & & -21878.5 & 0.0953 & 3 \\
\hline 48373.360 & 48373.3607 & $\mathrm{~s}$ & Vis & 0.004 & -20892.5 & 0.0875 & 3 \\
\hline 49345.583 & 49345.5837 & $\mathrm{~s}$ & Vis & 0.005 & -18266.5 & 0.0814 & 3 \\
\hline 49773.920 & 49773.9207 & $\mathrm{~s}$ & Vis & 0.008 & -17109.5 & 0.0600 & 3 \\
\hline 52546.3492 & 52546.3500 & $\mathrm{p}$ & CCD & & -9621 & 0.0070 & 4 \\
\hline 52546.5368 & 52546.5375 & $\mathrm{~s}$ & CCD & & -9620.5 & 0.0095 & 4 \\
\hline 53524.1272 & 53524.1279 & $\mathrm{p}$ & CCD & & -6980 & 0.0024 & 4 \\
\hline 53524.3073 & 53524.3080 & $\mathrm{~s}$ & CCD & & -6979.5 & -0.0027 & 4 \\
\hline 53860.4807 & 53860.4814 & $\mathrm{~s}$ & CCD & 0.0002 & -6071.5 & 0.0001 & 5 \\
\hline 53862.3329 & 53862.3327 & $\mathrm{~s}$ & $\mathrm{CCD}$ & 0.0002 & -6066.5 & 0.0002 & 5 \\
\hline 53863.2572 & 53863.2579 & $\mathrm{p}$ & CCD & 0.0002 & -6064 & -0.0002 & 5 \\
\hline
\end{tabular}


Table 2 continued

\begin{tabular}{|c|c|c|c|c|c|c|c|}
\hline $\begin{array}{l}\text { HJD } \\
(2400000+)\end{array}$ & $\begin{array}{l}\text { BJD } \\
(2400000+)\end{array}$ & $\overline{\mathrm{p} / \mathrm{s}}$ & Method & $\begin{array}{l}\begin{array}{l}\text { Error } \\
\text { (days) }\end{array}\end{array}$ & 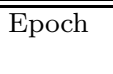 & $\begin{array}{l}O-C \\
\text { (days) }\end{array}$ & Ref. \\
\hline 53863.4424 & 53863.4432 & $\mathrm{~s}$ & CCD & 0.0002 & -6063.5 & 0 & 5 \\
\hline 53864.3674 & 53864.3682 & $\mathrm{p}$ & CCD & 0.0002 & -6061 & -0.0006 & 5 \\
\hline 53870.2910 & 53870.2918 & $\mathrm{p}$ & CCD & 0.0002 & -6045 & -0.0007 & 5 \\
\hline 53880.2876 & 53880.2884 & $\mathrm{p}$ & CCD & 0.0002 & -6018 & -0.0004 & 5 \\
\hline 53881.3981 & 53881.3989 & $\mathrm{p}$ & CCD & 0.0002 & -6015 & -0.0006 & 5 \\
\hline 53886.3971 & 53886.3978 & $\mathrm{~s}$ & CCD & 0.0002 & -6001.5 & 0.0003 & 5 \\
\hline 53887.3216 & 53887.3223 & $\mathrm{p}$ & $\mathrm{CCD}$ & 0.0003 & -5999 & -0.0008 & 5 \\
\hline 53888.2482 & 53888.2489 & $\mathrm{~s}$ & $\mathrm{CCD}$ & 0.0002 & -5996.5 & 0.0002 & 5 \\
\hline 53890.2835 & 53890.2842 & $\mathrm{p}$ & CCD & 0.0002 & -5991 & -0.0008 & 5 \\
\hline 53892.3205 & 53892.3212 & $\mathrm{~s}$ & CCD & 0.0002 & -5985.5 & -0.0001 & 5 \\
\hline 53896.3926 & 53896.3933 & $\mathrm{~s}$ & CCD & 0.0001 & -5974.5 & -0.0005 & 5 \\
\hline 53897.3175 & 53897.3183 & $\mathrm{p}$ & CCD & 0.0001 & -5972 & -0.0011 & 5 \\
\hline 53898.2438 & 53898.2445 & $\mathrm{~s}$ & CCD & 0.0002 & -5969.5 & -0.0005 & 5 \\
\hline 53902.3162 & 53902.3169 & $\mathrm{~s}$ & CCD & 0.0001 & -5958.5 & -0.0006 & 5 \\
\hline 53904.3517 & 53904.3524 & $\mathrm{p}$ & CCD & 0.0002 & -5953 & -0.0014 & 5 \\
\hline 53905.2782 & 53905.2789 & $\mathrm{~s}$ & CCD & 0.0002 & -5950.5 & -0.0005 & 5 \\
\hline 53907.3135 & 53907.3142 & $\mathrm{p}$ & CCD & 0.0002 & -5945 & -0.0015 & 5 \\
\hline 53910.2755 & 53910.2762 & $\mathrm{p}$ & CCD & 0.0002 & -5937 & -0.0013 & 5 \\
\hline 53917.3099 & 53917.3107 & $\mathrm{p}$ & $\mathrm{CCD}$ & 0.0003 & -5918 & -0.0013 & 5 \\
\hline 54132.5998 & 54132.6005 & $\mathrm{~s}$ & $\mathrm{CCD}$ & 0.0001 & -5336.5 & -0.0013 & 5 \\
\hline 54136.4867 & 54136.4875 & $\mathrm{p}$ & CCD & 0.0002 & -5326 & -0.0018 & 5 \\
\hline 54140.5594 & 54140.5601 & $\mathrm{p}$ & CCD & 0.0003 & -5315 & -0.0017 & 5 \\
\hline 54145.5585 & 54145.5592 & s & CCD & 0.0001 & -5301.5 & -0.0007 & 5 \\
\hline 54148.5203 & 54148.5211 & $\mathrm{~s}$ & CCD & 0.0002 & -5293.5 & -0.0007 & 5 \\
\hline 54151.4817 & 54151.4825 & $\mathrm{~s}$ & CCD & 0.0002 & -5285.5 & -0.0012 & 5 \\
\hline 54153.5172 & 54153.5180 & $\mathrm{p}$ & CCD & 0.0002 & -5280 & -0.0020 & 5 \\
\hline 54155.5552 & 54155.5559 & $\mathrm{~s}$ & CCD & 0.0002 & -5274.5 & -0.0003 & 5 \\
\hline 54159.4407 & 54159.4414 & $\mathrm{p}$ & CCD & 0.0002 & -5264 & -0.0023 & 5 \\
\hline 54168.5139 & 54168.5146 & $\mathrm{~s}$ & $\mathrm{CCD}$ & 0.0001 & -5239.5 & 0.0003 & 5 \\
\hline 54169.4369 & 54169.4376 & $\mathrm{p}$ & CCD & 0.0003 & -5237 & -0.0023 & 5 \\
\hline 54169.6248 & 54169.6256 & $\mathrm{~s}$ & $\mathrm{CCD}$ & 0.0002 & -5236.5 & 0.0005 & 5 \\
\hline 54170.3648 & 54170.3655 & $\mathrm{~s}$ & $\mathrm{CCD}$ & 0.0003 & -5234.5 & 0 & 5 \\
\hline 54170.5480 & 54170.5487 & $\mathrm{p}$ & CCD & 0.0003 & -5234 & -0.0019 & 5 \\
\hline 54175.3611 & 54175.3619 & $\mathrm{p}$ & CCD & 0.0003 & -5221 & -0.0018 & 5 \\
\hline 54176.4718 & 54176.4725 & $\mathrm{p}$ & CCD & 0.0002 & -5218 & -0.0018 & 5 \\
\hline 54178.3229 & 54178.3236 & $\mathrm{p}$ & CCD & 0.0003 & -5213 & -0.0018 & 5 \\
\hline 54179.6203 & 54179.6210 & $\mathrm{~s}$ & CCD & 0.0002 & -5209.5 & -0.0003 & 5 \\
\hline 54180.3606 & 54180.3613 & $\mathrm{~s}$ & CCD & 0.0002 & -5207.5 & -0.0004 & 5 \\
\hline 54180.5442 & 54180.5449 & $\mathrm{p}$ & CCD & 0.0002 & -5207 & -0.0019 & 5 \\
\hline 54181.4711 & 54181.4718 & $\mathrm{~s}$ & CCD & 0.0001 & -5204.5 & -0.0006 & 5 \\
\hline 54183.3222 & 54183.3229 & $\mathrm{~s}$ & CCD & 0.0001 & -5199.5 & -0.0007 & 5 \\
\hline 54184.4333 & 54184.4340 & $\mathrm{~s}$ & CCD & 0.0002 & -5196.5 & -0.0003 & 5 \\
\hline 54199.4263 & 54199.4271 & $\mathrm{p}$ & $\mathrm{CCD}$ & 0.0002 & -5156 & -0.0016 & 5 \\
\hline 54200.3521 & 54200.3528 & $\mathrm{~s}$ & CCD & 0.0002 & -5153.5 & -0.0015 & 5 \\
\hline 54200.5367 & 54200.5375 & $\mathrm{p}$ & CCD & 0.0002 & -5153 & -0.0019 & 5 \\
\hline 54201.2776 & 54201.2783 & $\mathrm{p}$ & CCD & 0.0002 & -5151 & -0.0016 & 5 \\
\hline 54203.3139 & 54203.3146 & $\mathrm{~s}$ & CCD & 0.0001 & -5145.5 & -0.0015 & 5 \\
\hline 54205.3500 & 54205.3507 & $\mathrm{p}$ & CCD & 0.0002 & -5140 & -0.0017 & 5 \\
\hline 54206.2758 & 54206.2765 & $\mathrm{~s}$ & CCD & 0.0002 & -5137.5 & -0.0014 & 5 \\
\hline 54207.3864 & 54207.3872 & $\mathrm{~s}$ & $\mathrm{CCD}$ & 0.0002 & -5134.5 & -0.0015 & 5 \\
\hline 54208.3115 & 54208.3122 & $\mathrm{p}$ & CCD & 0.0002 & -5132 & -0.0021 & 5 \\
\hline 54211.2732 & 54211.2740 & $\mathrm{p}$ & CCD & 0.0002 & -5124 & -0.0022 & 5 \\
\hline 54211.4592 & 54211.4599 & $\mathrm{~s}$ & CCD & 0.0002 & -5123.5 & -0.0013 & 5 \\
\hline 54214.4216 & 54214.4223 & $\mathrm{~s}$ & CCD & 0.0002 & -5115.5 & -0.0008 & 5 \\
\hline 54216.2724 & 54216.2731 & $\mathrm{~s}$ & CCD & 0.0001 & -5110.5 & -0.0011 & 5 \\
\hline 54225.3426 & 54225.3433 & $\mathrm{p}$ & CCD & 0.0002 & -5086 & -0.0016 & 5 \\
\hline 54231.2660 & 54231.2667 & $\mathrm{p}$ & CCD & 0.0002 & -5070 & -0.0019 & 5 \\
\hline 54232.3766 & 54232.3773 & $\mathrm{p}$ & CCD & 0.0002 & -5067 & -0.0020 & 5 \\
\hline 54233.3038 & 54233.3045 & $\mathrm{~s}$ & CCD & 0.0001 & -5064.5 & -0.0004 & 5 \\
\hline 54238.3003 & 54238.3011 & $\mathrm{p}$ & CCD & 0.0002 & -5051 & -0.0020 & 5 \\
\hline 54246.2616 & 54246.2623 & $\mathrm{~s}$ & CCD & 0.0002 & -5029.5 & -0.0007 & 5 \\
\hline 54254.2189 & 54254.2196 & $\mathrm{p}$ & CCD & 0.0003 & -5008 & -0.0034 & 5 \\
\hline 54254.4052 & 54254.4060 & $\mathrm{~s}$ & CCD & 0.0002 & -5007.5 & -0.0022 & 5 \\
\hline 54265.3273 & 54265.3281 & $\mathrm{p}$ & CCD & 0.0002 & -4978 & -0.0019 & 5 \\
\hline
\end{tabular}


X. Zhou et. al

Table 2 continued

\begin{tabular}{|c|c|c|c|c|c|c|c|}
\hline $\begin{array}{l}\text { HJD } \\
(2400000+)\end{array}$ & $\begin{array}{l}\text { BJD } \\
(2400000+)\end{array}$ & $\overline{\mathrm{p} / \mathrm{s}}$ & Method & $\begin{array}{l}\begin{array}{l}\text { Error } \\
(\text { days })\end{array} \\
\text { (d) }\end{array}$ & $\overline{\text { Epoch }}$ & $\begin{array}{l}O-C \\
\text { (days) }\end{array}$ & $\overline{\text { Ref. }}$ \\
\hline 54266.2535 & 54266.2543 & $\mathrm{~s}$ & CCD & 0.0001 & -4975.5 & -0.0013 & 5 \\
\hline 54268.2896 & 54268.2904 & $\mathrm{p}$ & CCD & 0.0002 & -4970 & -0.0015 & 5 \\
\hline 54271.2512 & 54271.2520 & $\mathrm{p}$ & CCD & 0.0002 & -4962 & -0.0017 & 5 \\
\hline 54273.2883 & 54273.2890 & $\mathrm{~s}$ & $\mathrm{CCD}$ & 0.0001 & -4956.5 & -0.0010 & 5 \\
\hline 54484.5038 & 54484.5045 & $\mathrm{p}$ & CCD & 0.0002 & -4386 & -0.0028 & 5 \\
\hline 54497.4625 & 54497.4632 & $\mathrm{p}$ & CCD & 0.0002 & -4351 & -0.0022 & 5 \\
\hline 54499.4993 & 54499.5000 & $\mathrm{~s}$ & $\mathrm{CCD}$ & 0.0001 & -4345.5 & -0.0017 & 5 \\
\hline 54502.4609 & 54502.4616 & s & CCD & 0.0002 & -4337.5 & -0.0020 & 5 \\
\hline 54525.4154 & 54525.4161 & $\mathrm{~s}$ & CCD & 0.0001 & -4275.5 & -0.0018 & 5 \\
\hline 54531.1528 & 54531.1536 & $\mathrm{p}$ & CCD & & -4260 & -0.0030 & 4 \\
\hline 54531.3398 & 54531.3406 & s & CCD & & -4259.5 & -0.0011 & 4 \\
\hline 54536.3364 & 54536.3372 & $\mathrm{p}$ & CCD & 0.0002 & -4246 & -0.0026 & 5 \\
\hline 54564.2897 & 54564.2904 & $\mathrm{~s}$ & CCD & 0.0001 & -4170.5 & -0.0019 & 5 \\
\hline 54566.3250 & 54566.3257 & $\mathrm{p}$ & $\mathrm{CCD}$ & 0.0002 & -4165 & -0.0029 & 5 \\
\hline 54569.2869 & 54569.2877 & $\mathrm{p}$ & CCD & 0.0002 & -4157 & -0.0028 & 5 \\
\hline 54571.3233 & 54571.3241 & $\mathrm{~s}$ & CCD & 0.0002 & -4151.5 & -0.0026 & 5 \\
\hline 54579.2827 & 54579.2835 & $\mathrm{p}$ & CCD & 0.0002 & -4130 & -0.0032 & 5 \\
\hline 54581.5041 & 54581.5048 & $\mathrm{p}$ & CCD & 0.0003 & -4124 & -0.0033 & 5 \\
\hline 54592.2414 & 54592.2422 & $\mathrm{p}$ & $\mathrm{CCD}$ & 0.0002 & -4095 & -0.0026 & 5 \\
\hline 54988.390 & 54988.3908 & $\mathrm{p}$ & CCD & 0.006 & -3025 & -0.0023 & 6 \\
\hline 55395.277 & 55395.2778 & $\mathrm{p}$ & CCD & 0.005 & -1926 & -0.0002 & 7 \\
\hline 56108.3440 & 56108.3448 & $\mathrm{p}$ & CCD & 0.0030 & 0 & 0 & 8 \\
\hline 57051.1458 & 57051.1466 & $\mathrm{~s}$ & CCD & 0.0002 & 2546.5 & 0.0061 & 4 \\
\hline 58224.6073 & 58224.6081 & $\mathrm{p}$ & CCD & 0.0001 & 5716 & 0.0173 & 9 \\
\hline 58224.7936 & 58224.7944 & $\mathrm{~s}$ & CCD & 0.0001 & 5716.5 & 0.0185 & 9 \\
\hline 58225.5337 & 58225.5345 & $\mathrm{~s}$ & CCD & 0.0001 & 5718.5 & 0.0181 & 9 \\
\hline 58225.7181 & 58225.7189 & $\mathrm{p}$ & $\mathrm{CCD}$ & 0.0001 & 5719 & 0.0174 & 9 \\
\hline 58226.6443 & 58226.6451 & $\mathrm{~s}$ & $\mathrm{CCD}$ & 0.0001 & 5721.5 & 0.0180 & 9 \\
\hline 58227.5692 & 58227.5700 & $\mathrm{p}$ & CCD & 0.0001 & 5724 & 0.0174 & 9 \\
\hline 58227.7551 & 58227.7559 & $\mathrm{~s}$ & CCD & 0.0001 & 5724.5 & 0.0181 & 9 \\
\hline 58228.4956 & 58228.4964 & $\mathrm{~s}$ & $\mathrm{CCD}$ & 0.0001 & 5726.5 & 0.0182 & 9 \\
\hline \multirow[t]{33}{*}{58228.6803} & 58228.6811 & $\mathrm{p}$ & CCD & 0.0001 & 5727 & 0.0178 & 9 \\
\hline & 58570.7804 & $\mathrm{p}$ & CCD & 0.0001 & 6651 & 0.0227 & 10 \\
\hline & 58570.9653 & $\mathrm{~s}$ & CCD & 0.0001 & 6651.5 & 0.0225 & 10 \\
\hline & 58571.1507 & $\mathrm{p}$ & CCD & 0.0001 & 6652 & 0.0227 & 10 \\
\hline & 58571.3356 & $\mathrm{~s}$ & CCD & 0.0001 & 6652.5 & 0.0225 & 10 \\
\hline & 58571.5209 & $\mathrm{p}$ & $\mathrm{CCD}$ & 0.0001 & 6653 & 0.0227 & 10 \\
\hline & 58571.7058 & $\mathrm{~s}$ & $\mathrm{CCD}$ & 0.0001 & 6653.5 & 0.0225 & 10 \\
\hline & 58571.8911 & $\mathrm{p}$ & CCD & 0.0001 & 6654 & 0.0227 & 10 \\
\hline & 58572.0761 & $\mathrm{~s}$ & $\mathrm{CCD}$ & 0.0001 & 6654.5 & 0.0226 & 10 \\
\hline & 58572.2614 & $\mathrm{p}$ & CCD & 0.0001 & 6655 & 0.0228 & 10 \\
\hline & 58572.4463 & $\mathrm{~s}$ & CCD & 0.0001 & 6655.5 & 0.0226 & 10 \\
\hline & 58572.8165 & $\mathrm{~s}$ & CCD & 0.0001 & 6656.5 & 0.0226 & 10 \\
\hline & 58573.0018 & $\mathrm{p}$ & CCD & 0.0001 & 6657 & 0.0228 & 10 \\
\hline & 58573.1867 & $\mathrm{~s}$ & CCD & 0.0001 & 6657.5 & 0.0225 & 10 \\
\hline & 58573.3720 & $\mathrm{p}$ & CCD & 0.0001 & 6658 & 0.0227 & 10 \\
\hline & 58573.5570 & $\mathrm{~s}$ & $\mathrm{CCD}$ & 0.0001 & 6658.5 & 0.0226 & 10 \\
\hline & 58573.7422 & $\mathrm{p}$ & CCD & 0.0001 & 6659 & 0.0227 & 10 \\
\hline & 58573.9273 & $\mathrm{~s}$ & $\mathrm{CCD}$ & 0.0001 & 6659.5 & 0.0226 & 10 \\
\hline & 58574.1125 & $\mathrm{p}$ & CCD & 0.0001 & 6660 & 0.0227 & 10 \\
\hline & 58574.2975 & $\mathrm{~s}$ & CCD & 0.0001 & 6660.5 & 0.0227 & 10 \\
\hline & 58574.4827 & $\mathrm{p}$ & CCD & 0.0001 & 6661 & 0.0227 & 10 \\
\hline & 58574.6678 & $\mathrm{~s}$ & CCD & 0.0001 & 6661.5 & 0.0227 & 10 \\
\hline & 58574.8529 & $\mathrm{p}$ & CCD & 0.0001 & 6662 & 0.0227 & 10 \\
\hline & 58575.0380 & $\mathrm{~s}$ & CCD & 0.0001 & 6662.5 & 0.0227 & 10 \\
\hline & 58575.2231 & $\mathrm{p}$ & CCD & 0.0001 & 6663 & 0.0226 & 10 \\
\hline & 58575.4083 & $\mathrm{~s}$ & CCD & 0.0001 & 6663.5 & 0.0227 & 10 \\
\hline & 58575.5933 & $\mathrm{p}$ & CCD & 0.0001 & 6664 & 0.0226 & 10 \\
\hline & 58575.7785 & s & $\mathrm{CCD}$ & 0.0001 & 6664.5 & 0.0227 & 10 \\
\hline & 58575.9636 & $\mathrm{p}$ & CCD & 0.0001 & 6665 & 0.0226 & 10 \\
\hline & 58576.1487 & $\mathrm{~s}$ & CCD & 0.0001 & 6665.5 & 0.0227 & 10 \\
\hline & 58576.3338 & $\mathrm{p}$ & $\mathrm{CCD}$ & 0.0001 & 6666 & 0.0226 & 10 \\
\hline & 58576.5189 & $\mathrm{~s}$ & CCD & 0.0001 & 6666.5 & 0.0226 & 10 \\
\hline & 58576.7040 & $\mathrm{p}$ & $\mathrm{CCD}$ & 0.0001 & 6667 & 0.0226 & 10 \\
\hline
\end{tabular}


Table 2 continued

\begin{tabular}{|c|c|c|c|c|c|c|c|}
\hline $\begin{array}{l}\text { HJD } \\
(2400000+)\end{array}$ & $\begin{array}{l}\text { BJD } \\
(2400000+)\end{array}$ & $\overline{\mathrm{p} / \mathrm{s}}$ & Method & $\begin{array}{l}\begin{array}{l}\text { Error } \\
(\text { days })\end{array} \\
\text { (d) }\end{array}$ & $\overline{\text { Epoch }}$ & $\begin{array}{l}O-C \\
\text { (days) }\end{array}$ & $\overline{\text { Ref. }}$ \\
\hline & 58576.8891 & $\mathrm{~s}$ & CCD & 0.0001 & 6667.5 & 0.0220 & 10 \\
\hline & 58577.0743 & $\mathrm{p}$ & CCD & 0.0001 & 6668 & 0.0226 & 10 \\
\hline & 58577.2594 & $\mathrm{~s}$ & CCD & 0.0001 & 6668.5 & 0.0226 & 10 \\
\hline & 58577.4445 & $\mathrm{p}$ & $\mathrm{CCD}$ & 0.0001 & 6669 & 0.0227 & 10 \\
\hline & 58577.6296 & $\mathrm{~s}$ & CCD & 0.0001 & 6669.5 & 0.0226 & 10 \\
\hline & 58577.8147 & $\mathrm{p}$ & CCD & 0.0001 & 6670 & 0.0226 & 10 \\
\hline & 58577.9998 & $\mathrm{~s}$ & CCD & 0.0001 & 6670.5 & 0.0226 & 10 \\
\hline & 58578.1850 & $\mathrm{p}$ & CCD & 0.0001 & 6671 & 0.0227 & 10 \\
\hline & 58578.3701 & s & CCD & 0.0001 & 6671.5 & 0.0226 & 10 \\
\hline & 58578.5552 & $\mathrm{p}$ & CCD & 0.0001 & 6672 & 0.0226 & 10 \\
\hline & 58578.7403 & s & CCD & 0.0001 & 6672.5 & 0.0226 & 10 \\
\hline & 58578.9255 & $\mathrm{p}$ & CCD & 0.0001 & 6673 & 0.0227 & 10 \\
\hline & 58579.1105 & $\mathrm{~s}$ & CCD & 0.0001 & 6673.5 & 0.0226 & 10 \\
\hline & 58579.2956 & $\mathrm{p}$ & $\mathrm{CCD}$ & 0.0001 & 6674 & 0.0226 & 10 \\
\hline & 58579.4807 & $\mathrm{~s}$ & CCD & 0.0001 & 6674.5 & 0.0226 & 10 \\
\hline & 58579.6659 & $\mathrm{p}$ & CCD & 0.0001 & 6675 & 0.0227 & 10 \\
\hline & 58579.8509 & $\mathrm{~s}$ & CCD & 0.0001 & 6675.5 & 0.0226 & 10 \\
\hline & 58580.0361 & $\mathrm{p}$ & CCD & 0.0001 & 6676 & 0.0227 & 10 \\
\hline & 58580.2212 & $\mathrm{~s}$ & CCD & 0.0001 & 6676.5 & 0.0226 & 10 \\
\hline & 58580.4064 & $\mathrm{p}$ & CCD & 0.0001 & 6677 & 0.0227 & 10 \\
\hline & 58580.5915 & s & CCD & 0.0001 & 6677.5 & 0.0226 & 10 \\
\hline & 58580.7767 & $\mathrm{p}$ & CCD & 0.0001 & 6678 & 0.0227 & 10 \\
\hline & 58580.9617 & $\mathrm{~s}$ & CCD & 0.0001 & 6678.5 & 0.0227 & 10 \\
\hline & 58581.1469 & $\mathrm{p}$ & CCD & 0.0001 & 6679 & 0.0227 & 10 \\
\hline & 58581.3320 & $\mathrm{~s}$ & CCD & 0.0001 & 6679.5 & 0.0227 & 10 \\
\hline & 58581.5171 & $\mathrm{p}$ & CCD & 0.0001 & 6680 & 0.0227 & 10 \\
\hline & 58581.7022 & $\mathrm{~s}$ & $\mathrm{CCD}$ & 0.0001 & 6680.5 & 0.0227 & 10 \\
\hline & 58584.4793 & $\mathrm{p}$ & $\mathrm{CCD}$ & 0.0001 & 6688 & 0.0230 & 10 \\
\hline & 58584.6641 & $\mathrm{~s}$ & CCD & 0.0001 & 6688.5 & 0.0228 & 10 \\
\hline & 58584.8494 & $\mathrm{p}$ & CCD & 0.0001 & 6689 & 0.0229 & 10 \\
\hline & 58585.0343 & $\mathrm{~s}$ & CCD & 0.0001 & 6689.5 & 0.0227 & 10 \\
\hline & 58585.2197 & $\mathrm{p}$ & CCD & 0.0001 & 6690 & 0.0230 & 10 \\
\hline & 58585.4046 & s & $\mathrm{CCD}$ & 0.0001 & 6690.5 & 0.0227 & 10 \\
\hline & 58585.5900 & $\mathrm{p}$ & CCD & 0.0001 & 6691 & 0.0230 & 10 \\
\hline & 58585.7748 & $\mathrm{~s}$ & CCD & 0.0001 & 6691.5 & 0.0227 & 10 \\
\hline & 58585.9602 & $\mathrm{p}$ & CCD & 0.0001 & 6692 & 0.0230 & 10 \\
\hline & 58586.1446 & $\mathrm{~s}$ & $\mathrm{CCD}$ & 0.0001 & 6692.5 & 0.0223 & 10 \\
\hline & 58586.3304 & $\mathrm{p}$ & $\mathrm{CCD}$ & 0.0001 & 6693 & 0.0230 & 10 \\
\hline & 58586.5152 & $\mathrm{~s}$ & CCD & 0.0001 & 6693.5 & 0.0227 & 10 \\
\hline & 58586.7007 & $\mathrm{p}$ & $\mathrm{CCD}$ & 0.0001 & 6694 & 0.0230 & 10 \\
\hline & 58586.8855 & $\mathrm{~s}$ & CCD & 0.0001 & 6694.5 & 0.0227 & 10 \\
\hline & 58587.0709 & $\mathrm{p}$ & CCD & 0.0001 & 6695 & 0.0230 & 10 \\
\hline & 58587.2558 & $\mathrm{~s}$ & CCD & 0.0001 & 6695.5 & 0.0228 & 10 \\
\hline & 58587.4411 & $\mathrm{p}$ & $\mathrm{CCD}$ & 0.0001 & 6696 & 0.0230 & 10 \\
\hline & 58587.6260 & $\mathrm{~s}$ & CCD & 0.0001 & 6696.5 & 0.0228 & 10 \\
\hline & 58587.8114 & $\mathrm{p}$ & CCD & 0.0001 & 6697 & 0.0230 & 10 \\
\hline & 58587.9962 & $\mathrm{~s}$ & $\mathrm{CCD}$ & 0.0001 & 6697.5 & 0.0227 & 10 \\
\hline & 58588.1816 & $\mathrm{p}$ & CCD & 0.0001 & 6698 & 0.0230 & 10 \\
\hline & 58588.3664 & $\mathrm{~s}$ & $\mathrm{CCD}$ & 0.0001 & 6698.5 & 0.0227 & 10 \\
\hline & 58588.5519 & $\mathrm{p}$ & CCD & 0.0001 & 6699 & 0.0230 & 10 \\
\hline & 58588.7367 & $\mathrm{~s}$ & CCD & 0.0001 & 6699.5 & 0.0227 & 10 \\
\hline & 58588.9221 & $\mathrm{p}$ & CCD & 0.0001 & 6700 & 0.0231 & 10 \\
\hline & 58589.1069 & $\mathrm{~s}$ & CCD & 0.0001 & 6700.5 & 0.0228 & 10 \\
\hline & 58589.2923 & $\mathrm{p}$ & CCD & 0.0001 & 6701 & 0.0230 & 10 \\
\hline & 58589.4768 & $\mathrm{~s}$ & CCD & 0.0001 & 6701.5 & 0.0224 & 10 \\
\hline & 58589.6625 & $\mathrm{p}$ & CCD & 0.0001 & 6702 & 0.0230 & 10 \\
\hline & 58589.8474 & $\mathrm{~s}$ & CCD & 0.0001 & 6702.5 & 0.0228 & 10 \\
\hline & 58590.0328 & $\mathrm{p}$ & CCD & 0.0001 & 6703 & 0.0230 & 10 \\
\hline & 58590.2176 & $\mathrm{~s}$ & $\mathrm{CCD}$ & 0.0001 & 6703.5 & 0.0228 & 10 \\
\hline & 58590.4031 & $\mathrm{p}$ & $\mathrm{CCD}$ & 0.0001 & 6704 & 0.0231 & 10 \\
\hline & 58590.5878 & $\mathrm{~s}$ & CCD & 0.0001 & 6704.5 & 0.0227 & 10 \\
\hline & 58590.7733 & $\mathrm{p}$ & $\mathrm{CCD}$ & 0.0001 & 6705 & 0.0231 & 10 \\
\hline & 58590.9580 & $\mathrm{~s}$ & CCD & 0.0001 & 6705.5 & 0.0227 & 10 \\
\hline & 58591.1435 & $\mathrm{p}$ & $\mathrm{CCD}$ & 0.0001 & 6706 & 0.0231 & 10 \\
\hline
\end{tabular}




\begin{tabular}{|c|c|c|c|c|c|c|c|}
\hline $\begin{array}{l}\text { HJD } \\
(2400000+)\end{array}$ & $\begin{array}{l}\text { BJD } \\
(2400000+)\end{array}$ & $\overline{\mathrm{p} / \mathrm{s}}$ & $\overline{\text { Method }}$ & $\begin{array}{l}\begin{array}{l}\text { Error } \\
\text { (days) }\end{array}\end{array}$ & Epoch & $\begin{array}{l}O-C \\
\text { (days) }\end{array}$ & $\overline{\text { Ref }}$ \\
\hline & 58591.3283 & $\mathrm{~s}$ & CCD & 0.0001 & 6706.5 & 0.0227 & 10 \\
\hline & 58591.5138 & $\mathrm{p}$ & $\mathrm{CCD}$ & 0.0001 & 6707 & 0.0231 & 10 \\
\hline & 58591.6985 & $\mathrm{~s}$ & CCD & 0.0001 & 6707.5 & 0.0227 & 10 \\
\hline & 58591.8840 & $\mathrm{p}$ & $\mathrm{CCD}$ & 0.0001 & 6708 & 0.0231 & 10 \\
\hline & 58592.0687 & $\mathrm{~s}$ & CCD & 0.0001 & 6708.5 & 0.0227 & 10 \\
\hline & 58592.2543 & $\mathrm{p}$ & CCD & 0.0001 & 6709 & 0.0232 & 10 \\
\hline & 58592.4389 & $\mathrm{~s}$ & $\mathrm{CCD}$ & 0.0001 & 6709.5 & 0.0227 & 10 \\
\hline & 58592.6245 & $\mathrm{p}$ & $\mathrm{CCD}$ & 0.0001 & 6710 & 0.0232 & 10 \\
\hline & 58592.8092 & $\mathrm{~s}$ & $\mathrm{CCD}$ & 0.0001 & 6710.5 & 0.0227 & 10 \\
\hline & 58592.9948 & $\mathrm{p}$ & $\mathrm{CCD}$ & 0.0001 & 6711 & 0.0232 & 10 \\
\hline & 58593.1794 & $\mathrm{~s}$ & $\mathrm{CCD}$ & 0.0001 & 6711.5 & 0.0227 & 10 \\
\hline & 58593.3650 & $\mathrm{p}$ & CCD & 0.0001 & 6712 & 0.0232 & 10 \\
\hline & 58593.5497 & $\mathrm{~s}$ & CCD & 0.0001 & 6712.5 & 0.0228 & 10 \\
\hline & 58593.7352 & $\mathrm{p}$ & $\mathrm{CCD}$ & 0.0001 & 6713 & 0.0232 & 10 \\
\hline & 58593.9199 & $\mathrm{~s}$ & $\mathrm{CCD}$ & 0.0001 & 6713.5 & 0.0228 & 10 \\
\hline & 58594.1055 & $\mathrm{p}$ & CCD & 0.0001 & 6714 & 0.0232 & 10 \\
\hline & 58594.2902 & $\mathrm{~s}$ & $\mathrm{CCD}$ & 0.0001 & 6714.5 & 0.0228 & 10 \\
\hline & 58594.4757 & $\mathrm{p}$ & CCD & 0.0001 & 6715 & 0.0231 & 10 \\
\hline & 58594.6605 & $\mathrm{~s}$ & CCD & 0.0001 & 6715.5 & 0.0228 & 10 \\
\hline & 58594.8460 & $\mathrm{p}$ & $\mathrm{CCD}$ & 0.0001 & 6716 & 0.0232 & 10 \\
\hline & 58595.0307 & $\mathrm{~s}$ & $\mathrm{CCD}$ & 0.0001 & 6716.5 & 0.0228 & 10 \\
\hline & 58595.2162 & $\mathrm{p}$ & $\mathrm{CCD}$ & 0.0001 & 6717 & 0.0232 & 10 \\
\hline & 58595.4009 & $\mathrm{~s}$ & $\mathrm{CCD}$ & 0.0001 & 6717.5 & 0.0228 & 10 \\
\hline & 58595.5864 & $\mathrm{p}$ & $\mathrm{CCD}$ & 0.0001 & 6718 & 0.0231 & 10 \\
\hline 58642.6058 & 58642.6066 & $\mathrm{p}$ & CCD & 0.0001 & 6845 & 0.0239 & 9 \\
\hline
\end{tabular}

References: (1) Bond (1970); (2) Sisteró \& Castore de Sisteró (1973); (3) BBSAG Bulletin\&2; (4) Mallama \& Pavlov (2015); (5) SuperWASF; ; (6) Paschke (2009); (7) Paschke (2010); (8) Paschke (2013); (9) The present work; (10) TESS.

The $O-C$ values calculated with Equation 1 are displayed in Fig. 2 with solid circles. The solid circles are split into two parts since the period of V752 Cen seems to change suddenly around the year 2000. Its period was constant during the first 30 years after it was first reported by Bond (1970), as displayed in the upper panel in Fig. 2 . The new linear equation is:

$$
\operatorname{Min} . I(B J D)=2456108.2896( \pm 0.0002)+0^{d} .370225027( \pm 0.000000005) \times E \text {. }
$$

The second part of the $O-C$ values as shown in the lower part of Fig. 2 is increasing continuously from the year 2000 up to the present day. Thus, a quadratic term is superposed on the initial linear Equation 1, The new ephemeris is:

$$
\begin{aligned}
& \operatorname{Min.I}(B J D)=2456108.3443( \pm 0.0001)+0^{d} .370233636( \pm 0.000000004) \times E \\
& +2.56( \pm 0.02) \times 10^{-10} \times E^{2} .
\end{aligned}
$$

The fitting of the observed O - C data reveals that the period of V752 Cen changed suddenly around the year 2000. Since then, it has been increasing continuously at a rate of $d P / d t=+5.05 \times 10^{-7}$ day $\cdot$ year $r^{-1}\left(+0.044 s \cdot y e a r^{-1}\right)$. To illustrate the sudden period change more clearly, the $\mathrm{O}-\mathrm{C}$ curve for the whole time span is shown in Fig. 3 It should be mentioned that we have tried to fit the observed $\mathrm{O}$ - $\mathrm{C}$ data with other possibilities, for example, orbital motion of the contact binary in a long-period elliptical orbit. However, the latter possibility would require the presence of a star (or stars) with an implausibly high mass (masses), so it was dropped from further consideration.

\section{MODELING THE LIGHT CURVES}

The light curves were obtained in April, 1971. The newly observed light curves displayed in Fig. 1]were obtained in April, 2018. The two sets of light curves are modeled with the Wilson-Devinney (W-D) program (Wilson 2012; Wilson \& Van Hamme 2014). The light curves show that V752 Cen is a totally eclipsed W UMa system. Mode 3 for contact binary is chosen, and the q-search method is not necessary (Terrell \& Wilson 2005). The mean surface temperature is given as $T_{1}=6138 K$ in the Gaia DR2 with a typical error of 324K (Andrae et al. 2018). Sisteró \& Castore de Sisteró (1974) reported that the spectral type of the primary star is F8, corresponding to a temperature of $6250 \mathrm{~K}$ (Cox 2000). The spectral types derived from B - V and $\mathrm{J}-\mathrm{H}$ were G0 and F7, respectively. We adopted the temperature from the Gaia DR2 since the uncertainty of about $300 \mathrm{~K}$ in temperature will not significantly change parameters like mass ratio, orbital inclination and fill-out factor (Jiang et al. 2015;

2 https://www.astronomie.info/bbsag/bulletins.html

3 https://wasp.cerit-sc.cz/form 


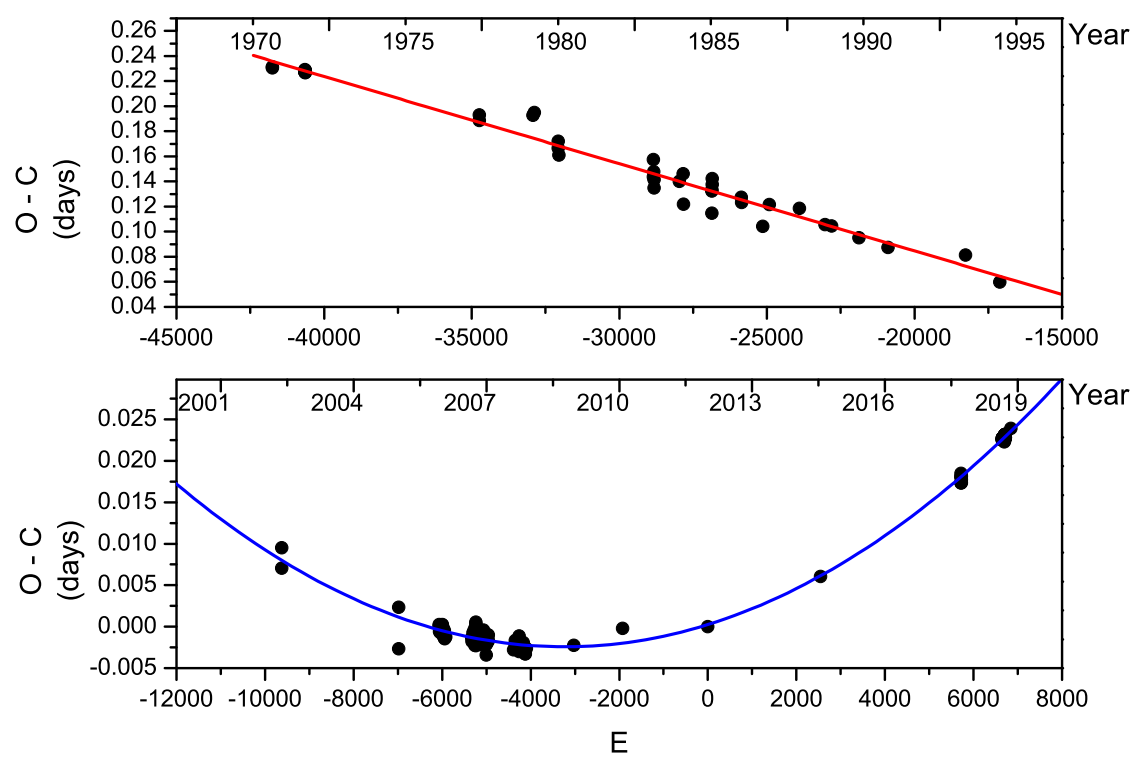

Figure 2. In both panels, the solid circles are O-C values calculated from Equation 1 In the upper panel, the red line represents, relative to Equation 1 the expected $\mathrm{O}-\mathrm{C}$ curve based on Equation 2 In the lower panel, the blue curve represents, relative to Equation 1 the expected O-C curve based on Equation 3

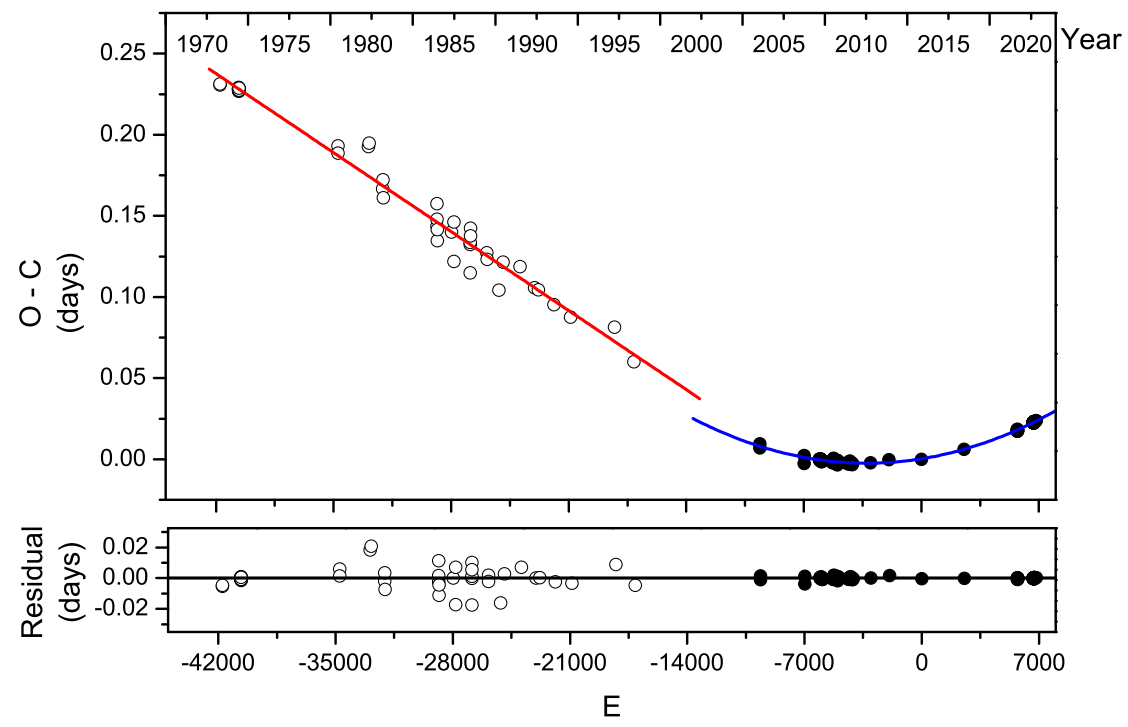

Figure 3. The O - C curve for the whole 48 - year interval.

Zhou et al. 2015). The gravity-darkening coefficients and bolometric albedo coefficients are set at $g_{1}=g_{2}=0.32($ Lucv 1967$)$ and $A_{1}=A_{2}=0.5$ (Ruciński 1969), respectively, and the limb darkening coefficients are set accordingly (Van Hamme 1993). The star eclipsed at primary minimum is assumed to be the primary star. The adjustable parameters are the mass ratio $(q)$, orbital inclination $(i)$, modified dimensionless surface potential of the primary star $\left(\Omega_{1}\right)$, mean surface temperature of the secondary star $\left(T_{2}\right)$, bandpass luminosities of the primary star $\left(L_{1}\right)$, and spots' parameters. The determined photometric parameters are listed in Table 3. The theoretical light curves based on the Roche model are displayed in Fig. 4 and Fig. 5 The $U$ and $B$ band light curves are shifted vertically by 0.3 and 0.1 mag in Fig. 4 . The $V$ band light curves observed on 19 April 2018 are shifted vertically by -0.2 mag in Fig. 5 . 
Table 3. Photometric solutions for V752 Cen

\begin{tabular}{lll}
\hline \hline Parameters & Data 1971 & Data 2018 \\
\hline$T_{1}(K)$ & $6138($ fixed $)$ & $6138($ fixed $)$ \\
$\mathrm{q}\left(M_{2} / M_{1}\right)$ & $3.31( \pm 0.02)$ & $3.35( \pm 0.01)$ \\
$i\left(^{\circ}\right)$ & $81.8( \pm 0.2)$ & $82.07( \pm 0.06)$ \\
$\Omega_{\text {in }}$ & 7.02 & 7.08 \\
$\Omega_{\text {out }}$ & 6.40 & 6.45 \\
$\Omega_{1}=\Omega_{2}$ & $6.85( \pm 0.02)$ & $6.89( \pm 0.01)$ \\
$T_{2}(K)$ & $5947( \pm 7)$ & $6014( \pm 2)$ \\
$\Delta T(K)$ & 191 & 124 \\
$T_{2} / T_{1}$ & $0.969( \pm 0.001)$ & $0.9798( \pm 0.0003)$ \\
$L_{1} /\left(L_{1}+L_{2}\right)(U)$ & $0.305( \pm 0.001)$ & \\
$L_{1} /\left(L_{1}+L_{2}\right)(B)$ & $0.296( \pm 0.001)$ & $0.2798( \pm 0.0003)$ \\
$L_{1} /\left(L_{1}+L_{2}\right)(V)$ & $0.286( \pm 0.001)$ & $0.2735( \pm 0.0003)$ \\
$L_{1} /\left(L_{1}+L_{2}\right)(V)$ & & $0.2735( \pm 0.0002)$ \\
$L_{1} /\left(L_{1}+L_{2}\right)\left(R_{c}\right)$ & & $0.2706( \pm 0.0003)$ \\
$L_{1} /\left(L_{1}+L_{2}\right)\left(I_{c}\right)$ & & $0.2683( \pm 0.0003)$ \\
$r_{1}($ pole $)$ & $0.2733( \pm 0.0005)$ & $0.2730( \pm 0.0003)$ \\
$r_{1}($ side $)$ & $0.2862( \pm 0.0006)$ & $0.2861( \pm 0.0003)$ \\
$r_{1}($ back $)$ & $0.3289( \pm 0.0011)$ & $0.3295( \pm 0.0004)$ \\
$r_{2}($ pole $)$ & $0.4664( \pm 0.0020)$ & $0.4676( \pm 0.0008)$ \\
$r_{2}($ side $)$ & $0.5045( \pm 0.0028)$ & $0.5059( \pm 0.0012)$ \\
$r_{2}($ back $)$ & $0.5336( \pm 0.0038)$ & $0.5351( \pm 0.0016)$ \\
$f$ & $27 \%( \pm 4 \%)$ & $29 \%( \pm 2 \%)$ \\
$\theta\left(^{\circ}\right)$ & $155.6( \pm 0.6)$ & $153.7( \pm 1.1)$ \\
$\psi\left({ }^{\circ}\right)$ & $240.5( \pm 2.7)$ & $278.6( \pm 0.5)$ \\
$r($ rad $)$ & $0.53($ fixed $)$ & $0.53($ fixed $)$ \\
$T_{f}$ & $0.73($ fixed $)$ & $0.73($ fixed $)$ \\
$\Sigma \omega(O-C)^{2}$ & 0.00256 & 0.00260 \\
\hline \hline
\end{tabular}

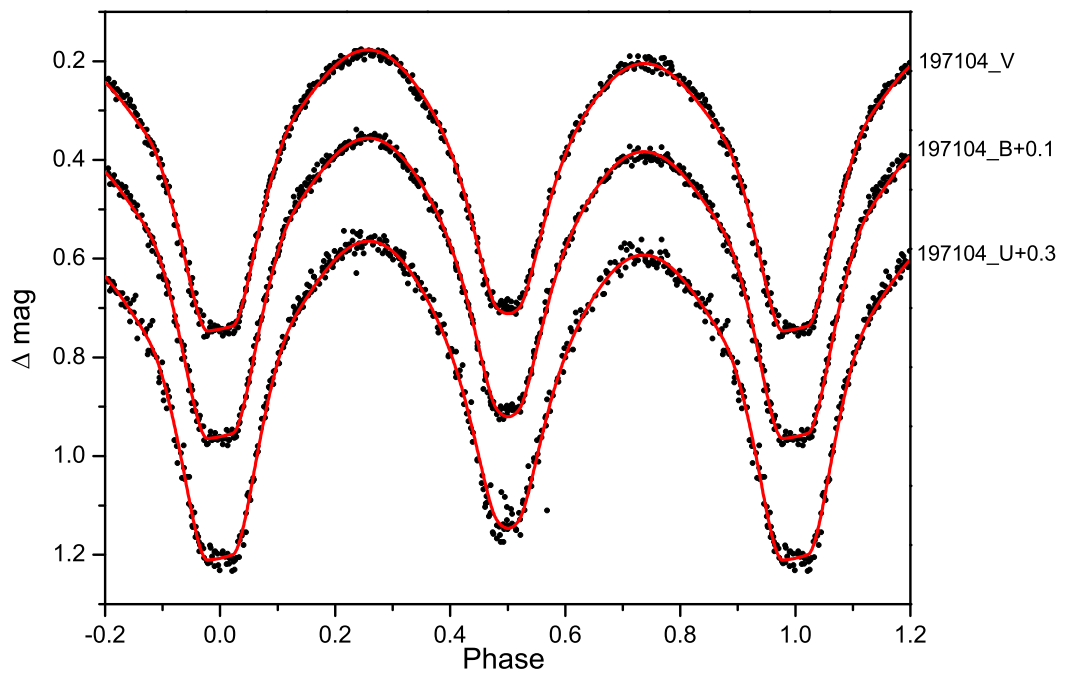

Figure 4. The solid circles are the $U, B$ and $V$ band light curves observed in April 1971 and the red lines are the corresponding theoretical light curves.

\section{DISCUSSION AND CONCLUSION}

According to the photometric solutions in Table 3, the light curves observed in 1971 and 2018 provide almost consistent results. The two component stars and geometric structure of V752 Cen were quite stable over the past forty-eight years. The solutions show that V752 Cen is a W-subtype contact binary sytem. The star eclipsed at primary minimum is hotter but less massive than the star eclipsed at secondary minimum. The orbital inclination is $i\left(^{\circ}\right)=82.07$, and the totally 


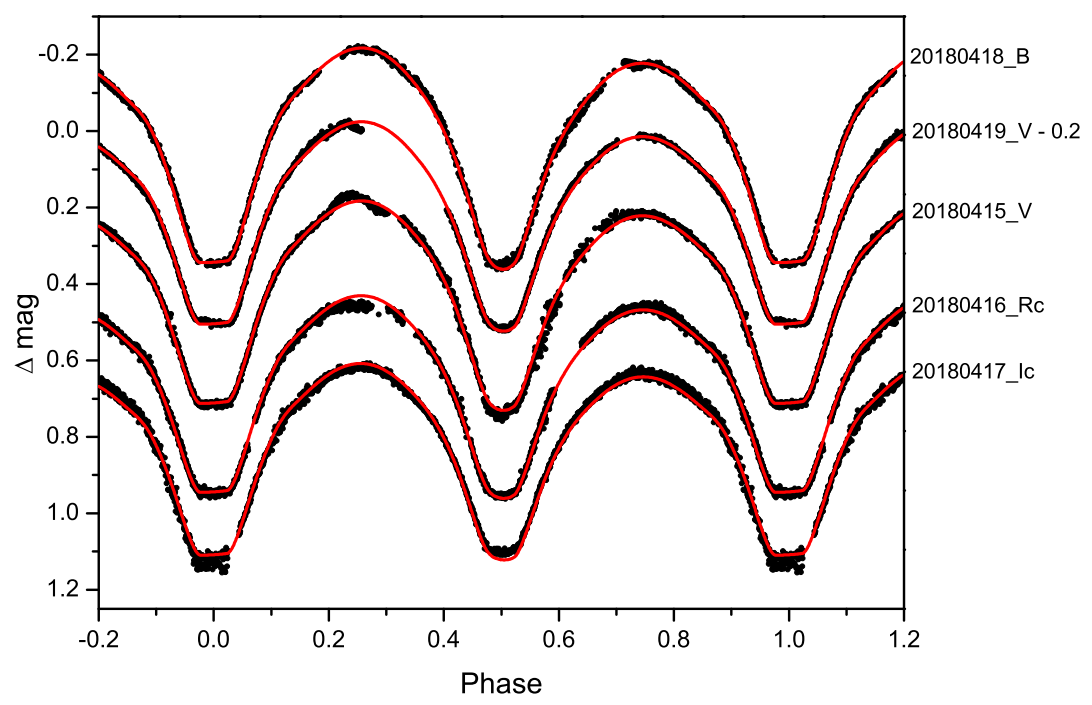

Figure 5. The solid circles are the $B, V, R_{c}$ and $I_{c}$ band light curves observed in April 2018 and the red lines are the corresponding theoretical light curves.

Table 4. Absolute parameters of components in V752 Cen

\begin{tabular}{lll}
\hline Parameters & Primary & Secondary \\
\hline$M$ & $0.39( \pm 0.02) M_{\odot}$ & $1.31( \pm 0.07) M_{\odot}$ \\
$R$ & $0.77( \pm 0.01) R_{\odot}$ & $1.30( \pm 0.02) R_{\odot}$ \\
$L$ & $0.75( \pm 0.03) L_{\odot}$ & $2.00( \pm 0.07) L_{\odot}$ \\
\hline
\end{tabular}

eclipsed characteristic implies that the determined parameters are very reliable. Considering the mass function obtained by Sisteró \& Castore de Sisteró (1974) $)\left(M_{1}+M_{2}\right) \sin ^{3} i=1.648 \pm 0.089 M_{\odot}$, the absolute parameters of the two component stars are calculated and listed in Table 4 . The orbital semi-major axis is calculated to be $a=2.59( \pm 0.05) R_{\odot}$. It should be mentioned that the mass ratio of V752 Cen was determined to be $q=0.31$ from spectroscopic observations (Sisteró \& Castore de Sisteró 1974), which was similar to our value $(1 / 3.35=0.30)$.

V752 Cen is a triple-lined spectroscopic quadruple system, and the tertiary and fourth components may make up a far away binary system (Schumacher 2009). We have also tried to set third light $\left(l_{3}\right)$ as a free parameter while modeling the light curve with the W - D program. However, the solution is not convergent. It means that the potential binary orbiting around V752 Cen should be late-type stars and don't contribute any light to the light curves of V752 Cen. The period analysis of V752 Cen reveals that its period was constant from 1970 to 2000, which means there was no mass transfer between the two component stars during that time interval. Then, its period changed suddenly around the year 2000. However, photometric analysis implies that the component stars in central binary system are very stable. Thus, the sudden period change may have been caused by the interaction between V752 Cen and the potential binary that is orbiting it. The period of V752 Cen has been increasing continuously at a rate of $d P / d t=+5.05 \times 10^{-7}$ day $\cdot$ year ${ }^{-1}\left(+0.044 s \cdot y e a r^{-1}\right)$ after that sudden period change happened. The period change is almost the same as that determined by Lohr et al. (2015), which was $+0.04379 s \cdot y e a r^{-1}$. It can be explained by mass transfer from the less massive component star to the more massive one, and the mass transfer rate is estimated to be $\frac{d M_{2}}{d t}=2.52 \times 10^{-7} M_{\odot} /$ year.

Both spectroscopic and photometric observations have indicated that many contact binaries are accompanied by at least one additional companion (Pribulla \& Rucinski 2006; Rappaport et al. 2013). Therefore, hierarchical contact binary systems are probably common. What is more important is that all short period M-dwarf contact binaries may have additional companions since the time-scale of angular momentum loss for the components is too long (Stepień 2011). Naoz \& Fabrycky (2014) claim that the eccentric Kozai-Lidov mechanism may remove angular momentum from central binaries and result in tidal tightening of inner binaries in triple star systems. However, no observational evidence on dynamic interactions between inner binaries and additional companions was reported. In that respect, V752 Cen is a very interesting hierarchical contact binary system with significant research value. The research on its light curves and period variations suggests that dynamic interactions may have happened between the inner binary and companion stars orbiting it. However, more observations and 
analyses on hierarchical contact binary systems are still needed to investigate the dynamic mechanism between the central binary and its companion stars in detail.

\section{ACKNOWLEDGEMENTS}

We appreciate the valuable comments and suggestions from the anonymous referee. We are grateful to Professor Wayne Orchiston for improving the manuscript. This research was supported by the National Natural Science Foundation of China (Grant No. 11703080 and 11703082), the Joint Research Fund in Astronomy (Grant No. U1931101) under cooperative agreement between the National Natural Science Foundation of China and Chinese Academy of Sciences, and the Yunnan Natural Science Foundation (Grant No. 2018FB006 and 2016FB004). It was part of the research activities at the National Astronomical Research Institute of Thailand (Public Organization). This work has made use of data from the European Space Agency (ESA) mission Gaia, processed by the Gaia Data Processing and Analysis Consortium (DPAC). Funding for the DPAC has been provided by national institutions, in particular the institutions participating in the Gaia Multilateral Agreement. This paper makes use of data from the DR1 of the WASP data as provided by the WASP consortium, and the computing and storage facilities at the CERIT Scientific Cloud, reg. no. CZ.1.05/3.2.00/08.0144 which is operated by Masaryk University, Czech Republic.

\section{REFERENCES}

Andrae, R., Fouesneau, M., Creevey, O., et al. 2018, A\&A, 616, A8

Barone, F., di Fiore, L., Milano, L., \& Russo, G. 1993, ApJ, 407, 237

Butters, O. W., West, R. G., Anderson, D. R., et al. 2010, A\&A, 520, L10

Bond, H. E. 1970, PASP, 82, 1065

Cox, A. N. 2000, Allen's Astrophysical Quantities (4th ed.; NewYork: Springer)

D’Angelo, C., van Kerkwijk, M. H., \& Rucinski, S. M. 2006, AJ, 132, 650.

Gaia Collaboration, Prusti, T., de Bruijne, J. H. J., et al. 2016, A\&A, 595, A1

Gaia Collaboration, Brown, A. G. A., Vallenari, A., et al. 2018, A\&A, 616, A1

Graczyk, D., Soszyński, I., Poleski, R., et al. 2011, Acta Astron., 61, 103

Jayasinghe, T., Stanek, K. Z., Kochanek, C. S., et al. 2019, MNRAS, 486, 1907

Jiang, L.-Q., Qian, S.-B., Zhang, J., et al. 2015, AJ, 149, 169

Leung, K.-C. 1976, PASP, 88, 936

Li, K., Hu, S.-M., Guo, D.-F., et al. 2015, AJ, 149, 120

Lohr, M. E., Norton, A. J., Payne, S. G., et al. 2015, A\&A, 578, A136

Lucy, L. B. 1967, ZAp, 65, 89

Mallama, A., \& Pavlov, H. 2015, Journal of the American Association of Variable Star Observers (JAAVSO), 43, 38

Naoz, S., \& Fabrycky, D. C. 2014, ApJ, 793, 137

Paschke, A. 2009, Open European Journal on Variable Stars, 116, 1

Paschke, A. 2010, Open European Journal on Variable Stars, 130, 1

Paschke, A. 2013, Open European Journal on Variable Stars, 155, 1

Pribulla, T., \& Rucinski, S. M. 2006, AJ, 131, 2986

Prša, A., Batalha, N., Slawson, R. W., et al. 2011, AJ, 141, 83

Qian, S.-B., Liu, N.-P., Li, K., et al. 2013, ApJS, 209, 13

Qian, S.-B., Zhou, X., Zola, S., et al. 2014, AJ, 148, 79

Qian, S.-B., He, J.-J., Zhang, J., et al. 2017, Research in Astronomy and Astrophysics, 17, 87

Qian, S.-B., Zhang, J., He, J.-J., et al. 2018, ApJS, 235, 5

Rappaport, S., Deck, K., Levine, A., et al. 2013, ApJ, 768, 33

Ricker, G. R., Winn, J. N., Vanderspek, R., et al. 2015, Journal of Astronomical Telescopes, Instruments, and Systems, 1, 014003

Ruciński, S. M. 1969, AcA, 19, 245

Ruciński, S. M. 1973, Acta Astron., 23, 79

Rucinski, S. M. 1997, AJ, 113, 1112

Schumacher, H. 2009, The Eighth Pacific Rim Conference on Stellar Astrophysics: A Tribute to Kam-Ching Leung, 404, 199

Sisteró, R. F., \& Castore de Sisteró, M. E. 1974, AJ, 79, 391

Sisteró, R. F., \& Castore de Sisteró, M. E. 1973, AJ, 78, 413

Sisteró, R. F., \& Castore de Sisteró, M. E. 1971, Information Bulletin on Variable Stars, 576, 1

Slawson, R. W., Prša, A., Welsh, W. F., et al. 2011, AJ, 142, 160. 
Soszyński, I., Pawlak, M., Pietrukowicz, P., et al. 2016, Acta Astron., 66, 405

Stȩpień, K. 2011, Acta Astron., 61, 139

Terrell, D., \& Wilson, R. E. 2005, Ap\&SS, 296, 221

Tokovinin, A., Thomas, S., Sterzik, M., et al. 2006, A\&A, 450, 681

Tutukov, A. V., Dremova, G. N., \& Svechnikov, M. A. 2004, Astronomy Reports, 48, 219

Van Hamme, W. 1993, AJ, 106, 2096

Wilson, R. E. 2012, AJ, 144, 73

Wilson, R. E., \& Van Hamme, W. 2014, ApJ, 780, 151

Yıldız, M., \& Doğan, T. 2013, MNRAS, 430, 2029

Xiao, Z., Shengbang, Q., Binghe, H., et al. 2016, PASJ, 68, 102

York, D. G., Adelman, J., Anderson, J. E., et al. 2000, AJ, 120, 1579

Zhou, X., Qian, S.-B., Liao, W.-P., et al. 2015, AJ, 150, 83

Zhou, X., Qian, S., \& Zhang, B. 2017, PASJ, 69, 37

Zhu, L. Y., Qian, S. B., Liu, N. P., Liu, L., \& Jiang, L. Q. 2013, AJ, 145, 39 